\title{
Rhythmic Robot Arm Control Using Oscillators
}

\author{
Matthew M. Williamson \\ MIT AI Lab, 545 Technology Square, Cambridge, MA 02139 \\ http://www.ai.mit.edu/people/matt
}

\begin{abstract}
This paper presents an approach to robot arm control based on exploiting the dynamical properties of a simple oscillator circuit coupled to the joints of an arm. Using the same architecture, a wide variety of tasks can be achieved without any explicit modeling of the arm or its environment. The inherent properties of the oscillators give robustness to perturbations and changes in frequency. The approach is implemented on two compliant arms, and is demonstrated to swing pendulums at their natural frequencies, turn cranks and manipulate 'Slinky' toys.
\end{abstract}

\section{Introduction}

This paper presents an approach to robot arm control that is computationally simple, and provides robust performance in a wide variety of rhythmic tasks. The method uses a set of simple oscillator circuits to control the motion of individual joints of the arm. Rather than take commands from a central controller, the oscillators use information about the joint state (torque and angle) to adapt the frequency and phase of the joint motion. The final motion of the individual joints is thus dependent on the dynamics of the complete system. By coupling together the oscillators and the arm in different dynamical situations (when the arm is free, constrained, manipulating objects etc.) different tasks can be performed.

This allows a different approach to robot control; instead of modeling the dynamics and reasoning about them [8], the interaction between arm and oscillator dynamics is exploited to perform tasks easily. The oscillators exhibit a strong entrainment property, responding over a wide range of frequencies with a single set of parameters, making the overall system robust to changes in system properties. They also entrain very rapidly (normally within one cycle), making the system robust to perturbations.

The tasks that have presently been achieved us-

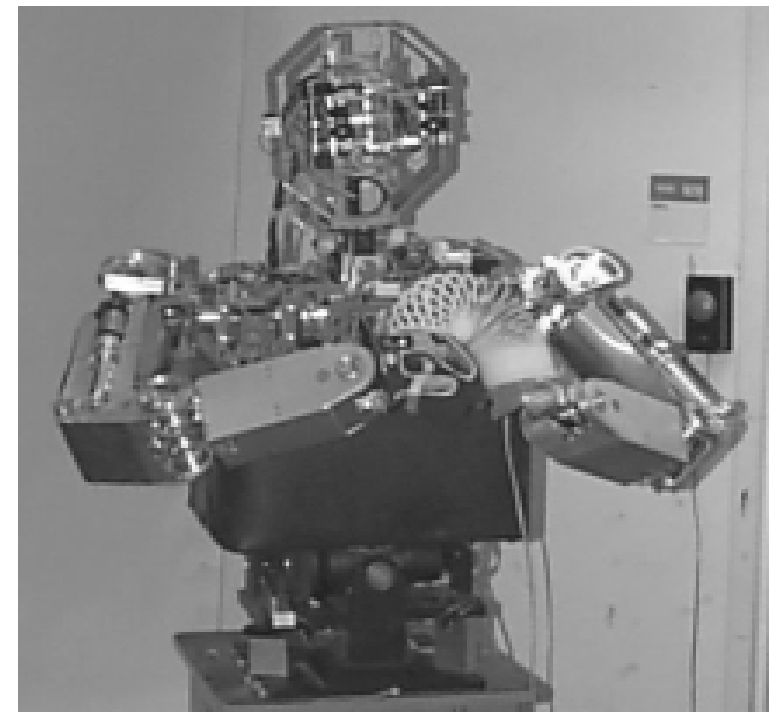

Figure 1: Cog playing with a 'Slinky' toy. This picture shows the humanoid form of the robot, with the two six degree of freedom arms used in this paper. The robot is using its elbow joints to move the slinky, exploiting the physical structure of the slinky to coordinate the two arms (see section 5).

ing this system include moving the arm at an efficient speed (automatically tuning into the resonant frequency of the system), exploiting position constraints to obtain coordinated multiple degree of freedom motion (turning a crank), and exploiting the force interactions between the arms and objects to perform rhythmic manipulation (playing with a 'Slinky' toy). The system is implemented on the arms of the humanoid robot Cog [2], which is shown in Figure 1.

This approach was inspired by the work of Greene [4], and also from consideration of how humans move their arms [1]. Similar approaches using neural oscillators have been taken by other researchers $[5,15]$, differing in complexity of the scheme, and the use in legs rather than arms. Robotic approaches exploiting rather than modeling dynamics have been suggested 
by Schaal [14], and McGeer [11].

The following sections describe the details of the oscillators and arms used, then examine in detail the properties of the oscillators in three different situations, indicating how they provide useful behavior. Conclusions and suggestions for further work are included at the end of the paper.

\section{The Arms and Oscillators}

The two six degree of freedom arms used in this work are mounted on the humanoid robot Cog [2] (shown in Figure 1. The arms are especially designed to interact stably and robustly with unstructured environments. They are lightweight and use series elastic actuators at every joint [12], which provide low noise force control, shock tolerance and stable interaction with passive environments [3].

At the joints of the arm, a simple proportionalderivative position control loop is used, making the torque at the $i$ th joint

$$
u_{i}=k_{i}\left(\theta_{v i}-\theta_{i}\right)-b_{i} \dot{\theta}_{i}
$$

where $k_{i}$ is the stiffness of the joint, $b_{i}$ the damping, $\theta_{i}$ the joint angle and $\theta_{v i}$ the equilibrium angle. The dynamic characteristics of the arm can be changed by altering the stiffness and damping, and the posture of the arm changed by altering the equilibrium angles. This type of joint level control is passive and so preserves stability of motion. Since the inner torque control is provided by series elastic actuators, the system is both compliant and shock resistant, making it suitable for operation in an unstructured environment.

The joints are controlled by a simple oscillatory circuit, consisting of two simulated neurons in mutual inhibition. The equations for the neurons are included below and describe the firing rate of real biological neurons [9], as might be found in a central pattern generator circuit.

$$
\begin{aligned}
\tau_{1} \dot{x}_{1} & =-x_{1}-\beta v_{1}-\omega y_{2}-\Sigma_{j=1}^{j=n} h_{j}\left[g_{j}\right]^{+}+c(2) \\
\tau_{2} \dot{v}_{1} & =-v_{1}+y_{1} \\
\tau_{1} \dot{x}_{2} & =-x_{2}-\beta v_{2}-\omega y_{1}-\Sigma_{j=1}^{j=n} h_{j}\left[g_{j}\right]^{-}+c(4) \\
\tau_{2} \dot{v}_{2} & =-v_{2}+y_{2} \\
y_{i} & =\left[x_{i}\right]^{+}=\max \left(x_{i}, 0\right) \\
y_{\text {out }} & =y_{1}-y_{2}
\end{aligned}
$$

where $x_{i}$ is the firing rate, $v_{i}$ is a variable representing the self-inhibition of the neuron (modulated by the adaptation constant $\beta$ ), and the mutual inhibition is controlled by the parameter $\omega^{1}$. The output of each neuron $y_{i}$ is taken as the positive part of $x_{i}$, and the output of the whole oscillator is $y_{\text {out }}$. The input $g_{j}$ is arranged to excite one neuron and inhibit the other, by applying the positive part $\left(\left[g_{j}\right]^{+}\right)$to one neuron and the negative part to the other. The inputs are scaled by gains $h_{j}$. The other important parameters are $c$, a constant that specifies the amplitude of the output, and two time constants $\tau_{1}$ and $\tau_{2}$. For steady oscillations, $\tau_{1} / \tau_{2}$ should be in the range $0.1-0.5$, the oscillator having an output frequency $w_{o s c} \propto 1 / \tau_{1}$. A detailed analysis of this type of oscillator was published in $[9,10]$.

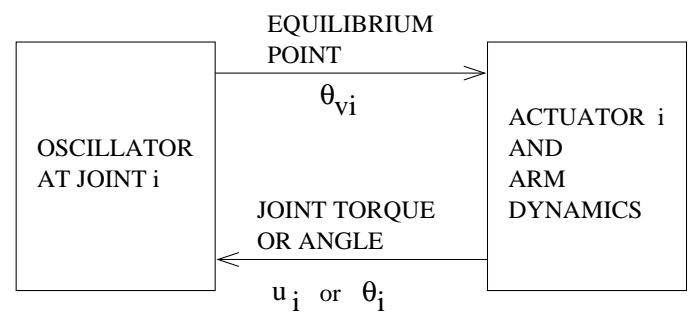

Figure 2: System schematic. The oscillator input is either the joint torque $u_{i}$ or the joint angle $\theta_{i}$, giving two different modes of operation. The output is used to drive the equilibrium angle $\theta_{v i}$ of the joint, and so move the arm. There is no set-point for the complete system, the system behavior emerging from the interaction between the oscillator and arm dynamics.

The oscillator is connected to the robot joints by using the output $y_{\text {out }}$ to move the equilibrium point $\theta_{v}$. The oscillations are about a fixed posture $\theta_{p}$, making the equilibrium point

$$
\theta_{v}=y_{1}-y_{2}+\theta_{p}=y_{\text {out }}+\theta_{p}
$$

For the examples in this paper, the inputs to the oscillators are taken to be either the force $\left(u_{i}\right)$ or the angle $\left(\theta_{i}\right)$ of the joint, as shown in Figure 2. These signals in general have an offset or DC component (due to gravity loading, or oscillation about a non-zero posture), which is removed using a high pass filter. The positive and negative parts of the resulting signal are then applied as the oscillator input. Figure 2 shows a schematic of the system. The oscillators and the actuators are tightly coupled; the system behavior emerges from the interaction of the oscillator, actuator, and arm dynamics. There is no control "set-point" for the joint motion, but by altering the time constants $\tau_{1}, \tau_{2}$, the amplitude constant $c$, and the type of feedback

\footnotetext{
${ }^{1}$ Values used: $\omega=2.5$ and $\beta=2.5$.
} 


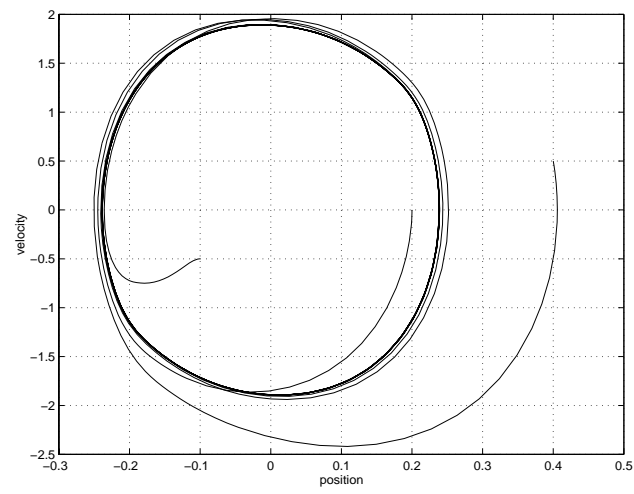

Figure 3: Phase plot of limb motion. This plot shows the velocity plotted against position for a single limb actuated by an oscillator using angle feedback. The limb is started with three different positions and velocities, and the system quickly converges to the limit cycle behavior. For this example $c=1.0, \tau_{1}=0.1, \tau_{2}=0.2, h_{1}=2$.

used ( $u$ or $\theta$ ) the behavior of the overall system can be modulated.

In the following three sections the behavior and usefulness of the oscillators is demonstrated in three cases: when the oscillators are driving a freely moving arm, when the arm is forced to move in a rhythmic path, and when a rhythmic force perturbation is applied to the arm. In each case the effect of using torque or angle feedback is analyzed, and an example of the robot performance is presented.

\section{$3 \quad$ Interaction with a free mass}

This section describes how the tight coupling of the oscillator and the arm can give efficient control of oscillatory motion, as well as providing robustness to changes in the overall system and to perturbations.

The behavior of the coupled oscillator-arm system when the actuated limb is free to move consists of oscillatory motion, the system converging quickly to a steady state frequency and amplitude of motion. This is illustrated by the phase plot of the limb motion from different starting conditions shown in Figure 3. The motion converges to the same pattern within at most half a cycle.

The frequency of the oscillation depends on the type of feedback used by the oscillator, as well as the mechanical properties of the system. If the limb is modeled as a simple mass, the resonant frequency of the system is $\omega_{\text {sys }}=\sqrt{k / m}$, where $k$ is the stiffness of the joint control (equation 8 ), and $m$ the mass. Figure 4 shows the steady state frequency of the mass oscillation for different feedback conditions, as the resonant frequency of the system is varied by changing $m$ and $k$. Under torque feedback, the system oscillates at a low frequency, and is not strongly dependent on the system resonant properties. However, by simply changing the feedback to use the joint angle, the steady state frequency ranges from being close to the oscillator natural frequency $w_{o s c}$ to frequencies close to $w_{\text {sys }}$.

Figure 5 shows the result of a single robot joint swinging a pendulum with variable natural frequency. Over the range of frequencies 5 to $9 \mathrm{rad} / \mathrm{s}$ (the oscillator natural frequency $w_{\text {osc }}$ is $7 \mathrm{rad} / \mathrm{s}$ ) the pendulum is swung at its natural frequency, demonstrating the robustness of the oscillator to changes in system dynamics.

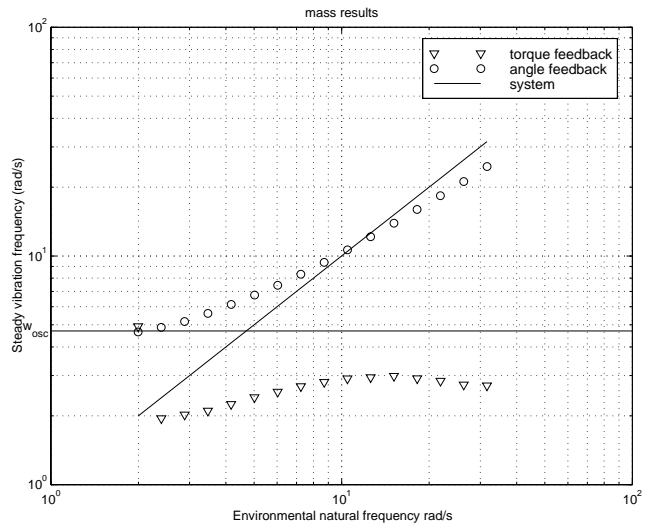

Figure 4: The response of the oscillator when connected to a free mass. The graph shows the steady state oscillation frequency obtained by simulating the system under different feedback conditions, and with different environmental resonant frequencies. The solid line indicates the system frequency. The triangles correspond to torque feedback, giving a low frequency response independent of the environmental conditions. The circles correspond to angle feedback, the frequency varying from the oscillator natural frequency $w_{\text {osc }}$ to frequencies close to resonance.

The natural frequency of a mechanical system is the most efficient speed from a control point of view, since only a small amount of energy need be injected to sustain the motion. The ability of the oscillators to tune into this frequency thus makes them useful for achieving efficient control. Since the oscillators can adapt to a range of different systems, they can respond to 


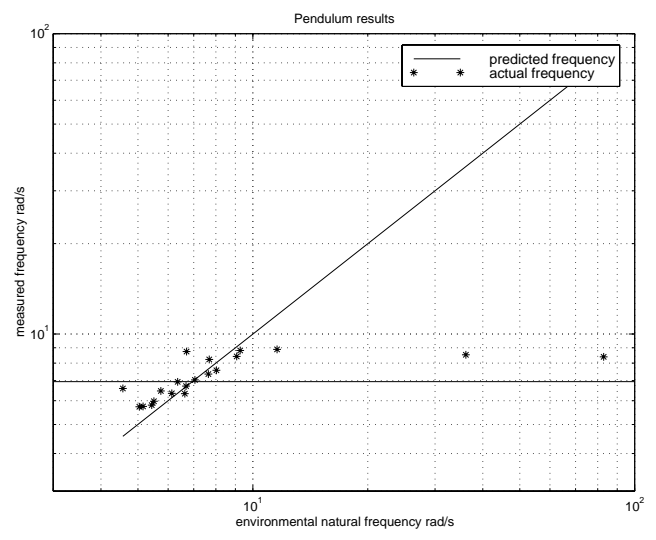

Figure 5: Performance of the robot swinging a pendulum. The graph shows the pendulum frequency when the oscillator is using angle feedback plotted against the natural frequency of the pendulum. The pendulum is swung at its natural frequency over the range 5 to $9 \mathrm{rad} / \mathrm{s}$. The natural frequency of the oscillator ( $w_{\text {osc }}$ - horizontal solid line) is $7 \mathrm{rad} / \mathrm{s}$ making the entrainment range about $60 \%$. The behavior at higher frequencies is most likely due to actuator bandwidth limitations.

changes in the dynamics of the system (such as when a mass is picked up) without requiring any parameter changes. The rejection of perturbations, as well as the reduced need to model the actuated system make this approach suitable for working in unstructured environments. Alternative approaches, of either determining the system frequencies by modeling the dynamics, or directly identifying the system [7] would also work, but could not respond so quickly to changes in the system dynamics.

\section{Interaction with position constraints}

This section explores the behavior of the oscillators when the motion of the actuated limb is imposed by some other system. This may occur from a human guiding the motion of the arm, or when the arm motion is constrained by a task, e.g., when turning a crank, the motion of individual robot joints are determined both by the crank motion, and the motion of the other joints. The adaptive nature of the oscillators presents opportunities of achieving coordination of a number of joints without requiring any communication between the joints, any central controller, or any explicit kinematic and dynamic models. This is because the oscillators can adapt their behavior to present either a small resisting force, or a force to aid the imposed motion. A further consequence of using the oscillators, is that these more complex tasks also display the robustness and perturbation rejection properties described in the previous section.
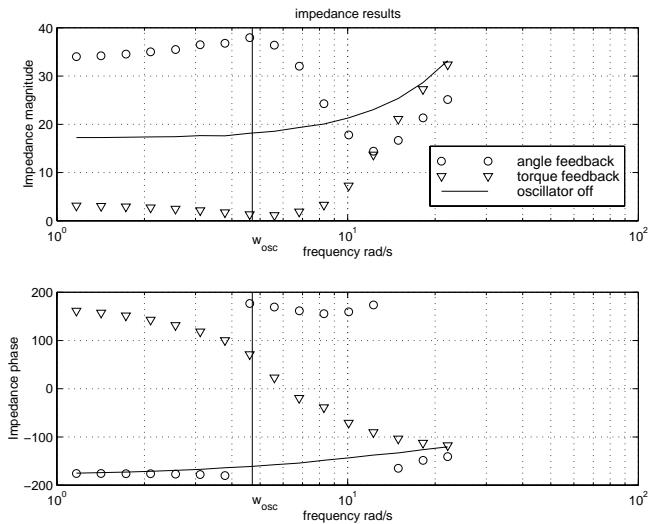

Figure 6: Output impedance of a single oscillator controlled joint. The upper and lower plots show the magnitude and phase of the impedance respectively, comparing torque feedback, angle feedback and no oscillator conditions. Phases greater than zero degrees indicate energy going out of the oscillator, and phases less than zero indicate energy being dissipated in the oscillator system. Under torque feedback (triangles), the magnitude of the impedance is low at frequencies less than $w_{o s c}$, the amount of energy going out of the oscillator reducing with frequency. Under angle feedback (circles), the oscillator strongly opposes the motion at low frequencies, but strongly aids it at frequencies just above $w_{o s c}$, reverting to a dissipative role at higher frequencies.

Imposing motion on a particular limb requires exerting a force to overcome the position control at the joint (equation 8). However if the equilibrium point is being altered by an oscillator, then the force required depends on the entrainment between the oscillator and the motion. For a wide range of frequencies, the oscillator can entrain to an imposed motion, moving the equilibrium point at the same frequency. The oscillator can also respond quickly to changes in the frequency of the motion. The relationship between the imposed motion and the force is defined as the mechanical impedance of the system. In general this cannot be calculated for a non-linear system, but since the force and the motion have the same frequency in this case, it can be calculated as a simple ratio. 
The impedance is often written as a complex number with a magnitude and phase, the phase determining whether the force is assisting or resisting the motion. Figure 6 shows how the impedance of the oscillator system varies with frequency.

Under torque feedback the impedance of the joint is reduced compared to the no oscillator condition at frequencies below the oscillator natural frequency $w_{\text {osc }}$. This small force assists the imposed motion. Under angle feedback, at low frequencies the oscillator acts to strongly resist the motion, while for frequencies above $w_{\text {osc }}$ the oscillator assists the motion.

This joint level behavior can make position constrained tasks such as crank turning easy. Instead of using a model to determine how to move each of the joints, the joints can adapt to the interaction between the arm motion and the crank motion. Attaching the arm to the crank, and driving one of the joints induces motion in the others. The oscillators can then adapt to the frequency of the induced motion, and change their impedance to assist the motion of the joint, so turning the crank. An example is shown in Figure 7, showing the coordination between the elbow and shoulder joints when angle feedback is used, and the resulting crank motion. Since the oscillators are highly adaptive, they can adapt to half-cycles as well as full ones. To facilitate complete crank motion, a small flywheel was added to the crank. In addition, it was found that while the system was settling into its stable state, the large internal forces created by uncoordinated joint action were forcing the arm away from stable states. By implementing a torque limit at the most powerful joint, the forces were reduced and smooth performance achieved. The stability of the crank turning is indicated by the speed in which the behavior is established and decays as the feedback is switched on and off.

The performance of the crank turning is robust to changes in frequency and to perturbations, returning to the stable crank motion. It is not robust to large changes in crank length or location, mainly because the oscillators operate around a fixed posture, with somewhat fixed amplitudes ${ }^{2}$. In addition, the system cannot handle very large cranks, because the sinusoidal-like outputs of the oscillators are not appropriate as joint commands for large motions. The torque limit mentioned above does allow the oscillators to produce a non-sinusoidal output, although in a rather limited way. Traditional robot approaches

\footnotetext{
${ }^{2}$ The actual amplitude of the oscillator output is partly determined by the constant $c$ (equation 2 ), and partly by the size of the feedback signal.
}
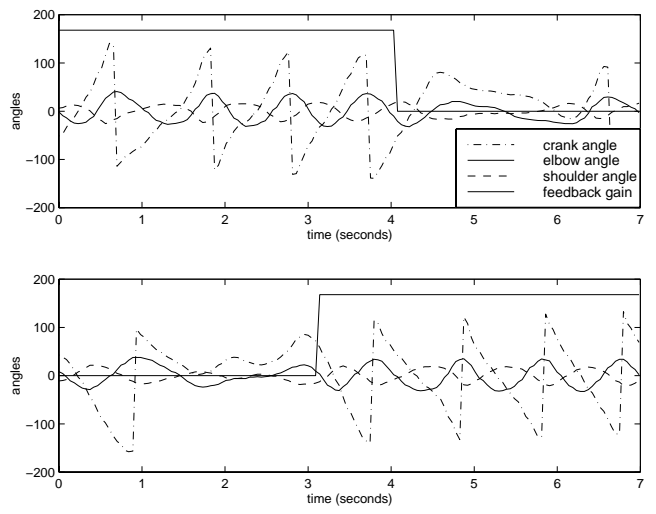

Figure 7: Angles of shoulder and elbow during crank turning. The angle of the crank is also illustrated (dash-dot), the saw tooth shape arises due to the position sensor for the crank wrapping around. The shoulder was driven at a constant frequency, and an oscillator with angle feedback was used at the elbow. When the feedback is on, the angles are coordinated and the crank is turned, otherwise there is no coordination.

to this kind of manipulation (hybrid force/position control [13], or impedance control [6]), use explicit kinematic knowledge of the arm and the crank location to solve this problem. Like the oscillator system, these methods are sensitive to errors in crank location. The advantage of using the oscillator method is that once the posture and approximate amplitudes have been specified, the other details of the system emerge from the interaction of the oscillators, the arm and the crank, without any further calculation. Another advantage is that no further calculation is needed to handle changes in crank frequency, or to deal with unexpected disturbances.

\section{$5 \quad$ Interaction with external forces}

This section explores the effect of external forces applied to the arm on the oscillator behavior. These forces could be exerted by manipulated objects, but also by humans guiding or pushing the arm. The tight coupling between the oscillator and the arm allows the oscillators to respond to these forces, so offering opportunities for the simple dynamic manipulation of objects. The useful properties of robustness to frequency changes and disturbances discussed previously can then be achieved in a different task situation.

As with the previous cases, the oscillator behavior 


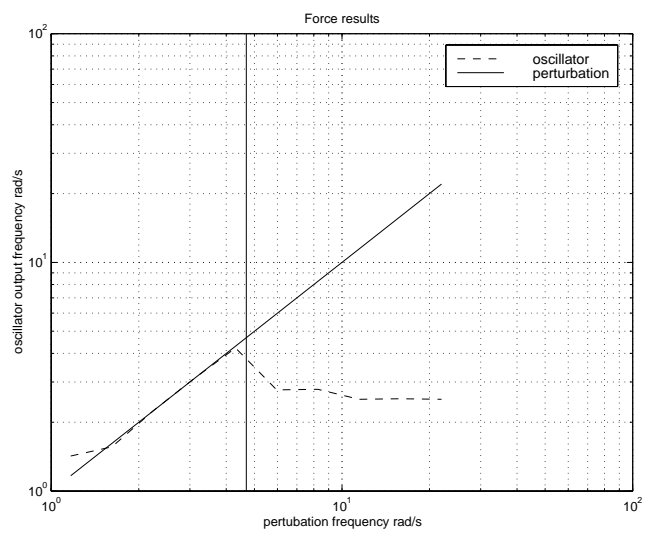

Figure 8: The response of the oscillator under a perturbing force. The graph shows the oscillator output frequency (dashed) and the perturbation frequency (solid) plotted against the perturbation frequency. It is only at frequencies below $w_{\text {osc }}$ (vertical line) that the oscillator is entrained (range 1.2 to $4 \mathrm{rad} / \mathrm{s}$ ).

differs under different feedback conditions. When angle feedback is used, the oscillator is insensitive to any force perturbations on the limb, itself a useful property. Under torque feedback, the oscillator can entrain the frequency of even a small force perturbation, and drive the joint at the same frequency (see Figure 8), although only for frequencies below $w_{o s c}$. Above $w_{o s c}$ the oscillator is not entrained.

This entrainment property has been used to manipulate a slinky toy, as shown in Figure 1. As the slinky is moved from hand to hand, the weight of the slinky in each hand is sensed and entrains the oscillators. The connection forces them to oscillate at the same frequency, and the motion of the weight forces them to oscillate out of phase. Figure 9 shows two examples of coordination using torque feedback.

It is obviously very simple to operate a slinky toy, it only requires the two arms to move out of phase with one another. However, using the oscillator system does provide some useful properties. As the oscillators can respond to imposed motions as well as forces, one can stop, start and interfere with the motion easily. If one of the hands is moved faster, the other speeds up to match it. If both hands are stopped and released, within one cycle the anti-phase motion is established. Interestingly, as well as the stable anti-phase motion, the system exhibits a less stable solution of moving both hands in phase. It is difficult to get this solution, since only a small asymmetry in the weight on the two hands results in the anti-phase motion.
As with the crank turning, a weakness of this approach is that the posture of the arm needs to be chosen by the user. This provides a place where traditional methods of choosing arm postures could mesh with the oscillator system. Since the communication is through the slinky, the best behavior is observed when the weight acts directly on the joint responsible for the motion. The advantage of designing the system in this way is the robustness and adaptability that the tightly coupled oscillator-arm system provides. The use of the slinky to coordinate the joints is very simple, and suggests that other coordination tasks might be achieved using naturally occurring forces. One example is exploiting the interaction forces between limb segments during motion for coordination. This has been achieved with this system, and is reported elsewhere [16].
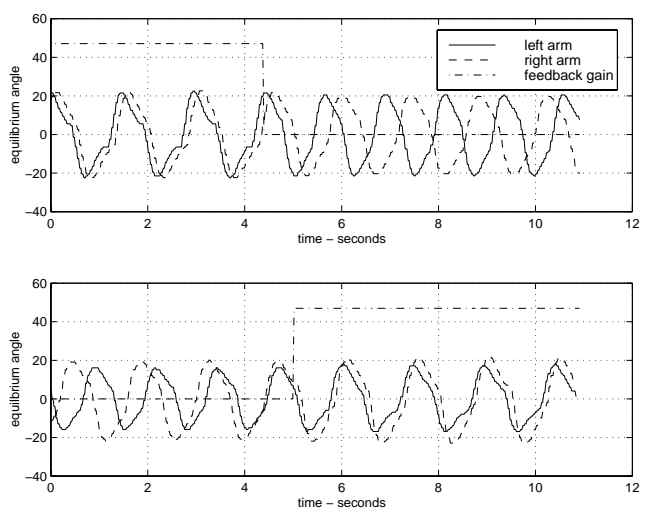

Figure 9: Two examples of slinky operation. Both plots show the outputs from the oscillators as the torque feedback (dashed) is turned on and off. When the traces are in phase, the slinky is moving in antiphase. When the feedback is on, the two arms are coordinated and the outputs are synchronized, but when off, the oscillators are no longer synchronized. The only connection between the oscillators is through the physical structure of the slinky.

\section{Discussion}

This paper has presented an approach to rhythmic robot-motion control that exploits the properties of a simple oscillator circuit to simplify computation, and obtain robust behavior in a variety of rhythmic tasks.

The oscillators converge rapidly onto a stable steady state solution, as was seen in the case of actuat- 
ing a mass, as well as in the crank and slinky coordination tasks. They can operate at a number of different frequencies without requiring changes in parameters, tuning into the resonant frequency of the pendulum motion, and also responding to speed changes in the crank turning and slinky. In addition to these properties, the oscillators can be used to achieve coordination between different robot joints, not through the use of explicit models, but though physical interaction with the arm dynamics. The physical structure of the arm and the crank, and the mass of the slinky were both used to produce the coordinated motion. By relying on the physics of the situation, the oscillator system can quickly respond to changes in the dynamics, which would otherwise be treated as a modeling error.

The oscillators are very simple, each having only two state variables, governed by a simple differential equation. The advantage that this proffers is tempered by the increasingly low cost of computation. However, using a simple tightly coupled scheme like this does reduce the possibility of numerical instabilities and calibration errors, which remain even when high speed computers are used.

One weakness of this approach is that the behavior is superimposed on top of a chosen arm posture. Choosing the correct posture is important to get the correct behavior (the slinky will not be operated correctly if it is held upside down!). This is a place where traditional robotic methods might be useful. The oscillators could handle the dynamic aspects of the task, while model based approaches deal with the arm gross motion and position.

In his paper on dynamic manipulation, Mason [8] commented that scaling a model-based approach to exploit the full dynamics of the arm was difficult. The same question is appropriate for this work, i.e., can this simple approach scale to more complex tasks? There are many avenues to be explored; more complex networks of oscillators, oscillators with more complex outputs (i.e., not sinusoidal), different systems of oscillators that can be switched in and out of control, and different feedback types, for example vision, audition, or touch sensing. The design of complex oscillators, and methods for tuning them is another area for research. The surprising ability of such a simple oscillator to perform the variety of coordinated tasks demonstrated in this paper certainly makes further investigation worthwhile.

\section{References}

[1] G. P. Bingham, R. C. Schmidt, M. T. Turvey, and
L. D. Rosenblum. Task dynamics and resource dynamics in the assembly of a coordinated rhythmic activity. Journal of Experimental Psychology: Human Perception and Performance, 17(2):359-381, 1991.

[2] R. A. Brooks and L. A. Stein. Building brains for bodies. Autonomous Robots, 1(1):7-25, 1994.

[3] E. Colgate and N. Hogan. An analysis of contact instability in terms of passive equivalents. In Proceedings of the 1989 IEEE International Conference on Robotics and Automation, pages 404-409, 1989.

[4] P. H. Greene. Why is it easy to control your arms? Journal of Motor Behavior, 14(4):260-286, 1982.

[5] N. G. Hatsopoulos, W. H. Warren, and J. N. Sanes. A neural pattern generator that tunes into the physical dynamics of the limb system. In Proceedings of the International Joint Conference on Neural Networks, volume 1, pages 104-109, Baltimore, MD, 1992.

[6] N. Hogan. Impedance control: An approach to manipulation. Journal of Dynamic Systems, Measurement, and Control, 107:1-24, 1985.

[7] L. Ljung. System identification : theory for the user. Prentice-Hall, 1987.

[8] M. T. Mason and K. M. Lynch. Dynamic manipulation. In Proceedings of the IEEE/RSJ International Workshop on Intelligent Robots and Systems (IROS93), pages 152-159, Yokohama, Japan, 1993.

[9] K. Matsuoka. Sustained oscillations generated by mutually inhibiting neurons with adaption. Biological Cybernetics, 52:367-376, 1985.

[10] K. Matsuoka. Mechanisms of frequency and pattern control in neural rhythm generators. Biological $\mathrm{Cy}$ bernetics, 56:345-353, 1987.

[11] T. McGeer. Passive walking with knees. In Proc 1990 IEEE Intl Conf on Robotics and Automation, 1990.

[12] G. A. Pratt and M. M. Williamson. Series elastic actuators. In Proceedings of the IEEE/RSJ International Conference on Intelligent Robots and Systems (IROS-95), volume 1, pages 399-406, Pittsburg, PA, July 1995.

[13] M. H. Raibert and J. J. Craig. Hybrid position/force control of manipulators. Journal of Dynamic Systems, Measurement, and Control, 103:126-133, 1981.

[14] S. Schaal and C. G. Atkeson. Open loop stable control strategies for robot juggling. In Proceedings 1993 IEEE International Conference on Robotics and Automation, volume 3, pages 913-918, 1993.

[15] G. Taga, Y. Yamaguchi, and H. Shimizu. Selforganized control of bipedal locomotion by neural oscillators in unpredictable environment. Biological Cybernetics, 65:147-159, 1991.

[16] M. Williamson. Exploiting natural dynamics in robot control. In Fourteenth European Meeting on Cybernetics and Systems Research (EMCSR '98), Vienna, Austria, 1998. 